\title{
Invasive liver abscess syndrome caused by Klebsiella pneumoniae with definite K2 serotyping in Japan: a case report
}

Ryota Seo ${ }^{1,2}$, Daisuke Kudo ${ }^{1,3 *}$, Yoshiaki Gu${ }^{4}$, Hisakazu Yano ${ }^{5}$, Tetsuji Aoyagi ${ }^{4}$, Taku Omura ${ }^{3}$, Shigemi Irino ${ }^{3,6}$, Mitsuo Kaku ${ }^{4}$ and Shigeki Kushimoto ${ }^{1,3}$

\begin{abstract}
Background: Klebsiella pneumonia is a well-known human pathogen, and recently, a distinct invasive syndrome caused by K. pneumoniae serotypes K1 and K2 has been recognized in Southeast Asia. This syndrome is characterized by primary liver abscess and extrahepatic complications resulting from bacteremic dissemination. We report the first adult case of primary liver abscess caused by the definite K2 serotyped pathogen, with endogenous endophthalmitis in Japan.

Case presentation: A 64-year-old woman was admitted to a nearby hospital for a high fever and diarrhea. She had visual loss of her right eye, renal dysfunction, and thrombocytopenia within $24 \mathrm{~h}$ from admission. She was transferred to our institution. On admission, she had no alteration of mental status and normal vital signs; however, she had almost complete ablepsia of the right eye. Laboratory data showed severe inflammation, liver dysfunction, thrombocytopenia, an increased serum creatinine level, and coagulopathy. Computed tomography showed a low density area in the right lobe of the liver. Invasive liver abscess syndrome probably caused by K. pneumonia was highly suspected and immediately administered broad-spectrum antibiotics for severe sepsis. Concurrently, endogenous endophthalmitis was diagnosed, and we performed vitrectomy on the day of admission. The blood culture showed $K$. pneumoniae infection. Percutaneous drainage of the liver abscess was also performed. Although she was discharged in a good general condition on day 22, she had complete ablepsia of the right eye. The K2A gene was detected by polymerase chain reaction (PCR), which is consistent with the K2 serotype. PCR was also positive for the virulenceassociated gene rmpA. Final diagnosis was invasive liver abscess syndrome caused by K2 serotype K. pneumonia.
\end{abstract}

Conclusions: Although the primary liver abscess caused by K. pneumoniae with a hypermucoviscous phenotype is infrequently reported outside Southeast Asia, physicians should recognize this syndrome, and appropriate diagnosis and treatment is essential for saving patients' lives and preserving organ function, especially for visual acuity.

Keywords: Klebsiella pneumoniae, Liver abscess, Endogenous endophthalmitis, K2 serotype

\footnotetext{
* Correspondence: kudodaisuke@med.tohoku.ac.jp

${ }^{1}$ Department of Emergency and Critical Care Medicine, Tohoku University

Hospital, 1-1 Seiryo-machi, Aoba-ku, Sendai 980-8574, Japan

${ }^{3}$ Division of Emergency and Critical Care Medicine, Tohoku University

Graduate School of Medicine, 2-1 Seiryo-machi, Aoba-ku, Sendai 980-8575,

Japan

Full list of author information is available at the end of the article
} 


\section{Background}

Klebsiella pneumoniae is a well-known human pathogen, and recently, a distinct invasive syndrome caused by $K$. pneumoniae serotypes $\mathrm{K} 1$ and $\mathrm{K} 2$ has been recognized in Southeast Asia, which is becoming an emerging disease worldwide [1].

The invasive nature of some $K$. pneumoniae strains include a hypermucoviscous phenotype associated with serotypes $\mathrm{K} 1$ and $\mathrm{K} 2$, and the regulator of the mucoid phenotype A gene (rmpA) [2]. Of those, K. pneumoniae strains expressing the capsular type $\mathrm{K} 1$ or $\mathrm{K} 2$ antigen have been reported to be especially virulent [3]. These serotypes have a high prevalence of resistance to phagocytosis, intracellular death by neutrophils, and bactericidal complements in a patient's serum [3].

This invasive syndrome has been reported in Southeast Asian countries [1]. However, only two adult cases caused by $K$. pneumoniae with definite serotyping of $\mathrm{K} 1[4,5]$ and one pediatric case with the $K 2$ serotype [6] have been reported in Japan.

Herein, we present the first adult case with invasive liver abscess syndrome and severe sepsis caused by $K$. pneumoniae with definite K2 serotyping, complicated with endogenous endophthalmitis resulting in complete ablepsia in a healthy woman in Japan.

\section{Case presentation}

A 64-year-old woman with an unremarkable medical history was admitted to a nearby hospital for a high fever and diarrhea that had lasted for 1 week. She had vision loss in her right eye, exacerbation of renal dysfunction (serum creatinine level, $4.96 \mathrm{mg} / \mathrm{dL}$ ), and thrombocytopenia (platelet count, 14,000/ $\mu \mathrm{L}$ ) within $48 \mathrm{~h}$ from initial presentation. Therefore, she was transferred to our institution for treatment of severe infectious disease. On admission, she was alert and had no alteration of mental status, no hypoxia on room air, and normal vital signs (temperature, $36.7{ }^{\circ} \mathrm{C}$; blood pressure, 126/80 mmHg; pulse rate, 74 beats/min; respiratory rate, 12 breaths/min). However, she had almost complete ablepsia of the right eye. Laboratory data showed severe inflammation (white cell count, $21,700 / \mu \mathrm{L}$; C-reactive protein level, $28.2 \mathrm{mg} / \mathrm{dL}$; procalcitonin, $46.4 \mathrm{ng} / \mathrm{mL}$ ), liver dysfunction (aspartate transaminase, $147 \mathrm{IU} / \mathrm{L}$; alanine transaminase, $345 \mathrm{IU} / \mathrm{L})$, thrombocytopenia (platelet count, $32,000 / \mu \mathrm{L}$ ), an increased serum creatinine level $(1.35 \mathrm{mg} / \mathrm{dL})$, and coagulopathy (fibrin/fibrinogen degradation product, $56.6 \mu \mathrm{g} / \mathrm{mL}$; D-dimer, $24.2 \mu \mathrm{g} /$ $\mathrm{mL}$; prothrombin time-international normalized ratio, 1.15)(Table 1). Computed tomography showed a low density area in the right lobe of the liver, and she was diagnosed with a liver abscess (Fig. 1a). Broad-spectrum antibiotic therapy with meropenem ( $3 \mathrm{~g} /$ day) was immediately started. Endogenous endophthalmitis of the right eye was also diagnosed, and emergency vitrectomy was performed on the day of admission. The blood culture at admission showed $K$. pneumoniae, and the antibiotic therapy was changed to ceftriaxone $(2 \mathrm{~g} /$ day $)$ on day 4 because all of the cephalosporin had high susceptibility (Table 2) and ceftriaxone was known to penetrate to the liver in high concentration, which was continued to day 14. Percutaneous drainage of the liver abscess, which was unresolved by medical treatment, was performed after coagulopathy improved on day 6 (Fig. 1b). The

Table 1 Changes in the patient's laboratory data

\begin{tabular}{|c|c|c|c|c|c|}
\hline & The previous hospital visit & At admission to our hospital & Day 3 & Day 6 & Reference ranges \\
\hline WBC count, $/ \mu \mathrm{L}$ & 22,900 & 21,700 & 14,100 & 13,400 & $4,000-9000$ \\
\hline $\mathrm{CRP}, \mathrm{mg} / \mathrm{dL}$ & 30.3 & 28.2 & 7.2 & 8.6 & $0.0-0.3$ \\
\hline$P C T, n g / m L$ & & 46.4 & 5.98 & 0.88 & $0.00-0.40$ \\
\hline T-Bil, mg/dL & 2.19 & 2 & 0.9 & 0.7 & $0.2-1.0$ \\
\hline AST, U/L & 206 & 147 & 24 & 25 & $8-38$ \\
\hline $\mathrm{ALT}, \mathrm{U} / \mathrm{L}$ & 423 & 345 & 96 & 62 & $4-43$ \\
\hline BUN, mg/dL & 57.1 & 49 & 13 & 14 & $8-20$ \\
\hline $\mathrm{Cr}, \mathrm{mg} / \mathrm{dL}$ & 1.73 & 1.35 & 0.66 & 0.55 & $0.44-1.15$ \\
\hline PLT count, $\times 10^{4} / \mu \mathrm{L}$ & 1.4 & 3.2 & 3.5 & 16.1 & $15-35$ \\
\hline PT-INR & & 1.2 & 1.08 & 1.15 & $<1.15$ \\
\hline APTT, s & & 34.5 & 28.8 & 30.8 & $29.6-40.8$ \\
\hline $\mathrm{FDP}, \mu \mathrm{g} / \mathrm{mL}$ & & 59.2 & 12.2 & 9.2 & $0.0-4.9$ \\
\hline D-dimer, $\mu \mathrm{g} / \mathrm{mL}$ & & 24.2 & 4.7 & 4.1 & $0.0-0.9$ \\
\hline
\end{tabular}

WBC white blood cell, CRP C-reactive protein, $P C T$ procalcitonin, $T$-Bil total bilirubin, $A S T$ aspartate transaminase, $A L T$ alanine transaminase, $B U N$ blood urea nitrogen, $C r$ creatinine, PLT platelet, PT-INR, prothrombin time-international normalized ratio, APTT activated partial thromboplastin time, FDP fibrin/fibrinogen degradation product 


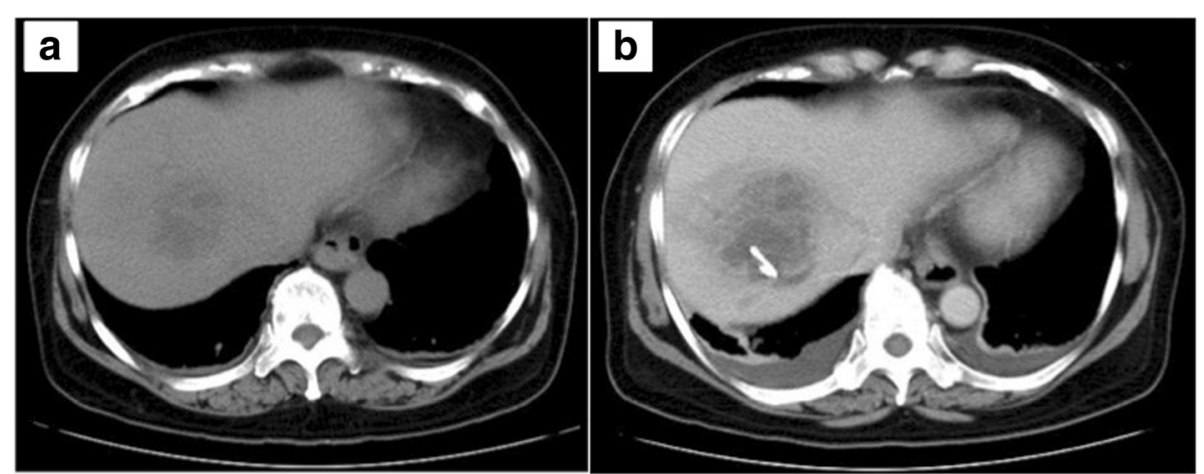

Fig. 1 Computed tomography scans. Plain computed tomography showing a low-density lesion in the right lobe of the liver at admission to our hospital (a). Enhanced computed tomography showing an unresolved liver abscess on day 6, which was drained percutaneously (b)

culture of the drainage fluid also showed K. pneumoniae. The drainage tube was removed on day 14 , and the patient was discharged in a good general condition on day 22, with an additional 2-week course of oral levofloxacin on days 14-28. However, she had complete ablepsia of the right eye. Capsular probing via PCR for the presence of magA (serotype K1) and K2A (serotype K2) genes was performed by using the magA-specific primers (forward, 5'-GGTGCTCTTTACATCATTGC-3'; and reverse, 5'GCAATGGCCATTTGCGTTAG- $\left.{ }^{\prime}\right)$ and $k 2 A$-specific primers (forward, 5'-CAACCATGGTGGTCGATTAG-3'; and reverse, 5' $^{\prime}$-TGGTAGCCATATCCCTTTGG-3') as

Table 2 Antimicrobial susceptibility of Klebsiella pneumoniae

\begin{tabular}{lll}
\hline Antimicrobial agent & MIC $(\mathrm{mg} / \mathrm{L})$ & MIC interpretation \\
\hline Ampicillin & 16 & $\mathrm{R}$ \\
Piperacillin & 8 & $\mathrm{R}$ \\
Cefazolin & $\leqq 4$ & $\mathrm{~S}$ \\
Cefotaxime & $\leqq 1$ & $\mathrm{~S}$ \\
Ceftazidime & $\leqq 1$ & $\mathrm{~S}$ \\
Imipenem/cilastatin & $\leqq 1$ & $\mathrm{~S}$ \\
Gentamicin & $\leqq 1$ & $\mathrm{~S}$ \\
Amikacin & $\leqq 2$ & $\mathrm{~S}$ \\
Minocycline & $\leqq 1$ & $\mathrm{~S}$ \\
Ciprofloxacin & $\leqq 0.25$ & $\mathrm{~S}$ \\
Sulfamethoxazole trimethoprim & $\leqq 20$ & $\mathrm{~S}$ \\
Cefepime & $\leqq 1$ & $\mathrm{~S}$ \\
Levofloxacin & $\leqq 0.12$ & $\mathrm{~S}$ \\
Meropenem & $\leqq 0.25$ & $\mathrm{~S}$ \\
Amoxicillin/clavulanic acid & $\leqq 2$ & $\mathrm{~S}$ \\
Cefmetazole & $\leqq 1$ & $\mathrm{~S}$ \\
Aztreonam & $\leqq 1$ & $\mathrm{~S}$ \\
Cefpodoxime & $\leqq 0.25$ & $\mathrm{~S}$ \\
\hline MIC minmum & &
\end{tabular}

MIC minimum inhibitory concentration, $S$ susceptible, $R$ resistant previously described[7]. The virulence-associated gene rmpA was also screened using PCR by using the $r m p A$ specific primers (forward, 5'- ACTGGGCTACCTCT GCTTCA-3'; and reverse, 5' - CTTGCATGAGCCAT CTTTCA-3') as previously described [8]. The reaction mixtures of these samples were kept at $95{ }^{\circ} \mathrm{C}$ for $5 \mathrm{~min}$, followed by 35 cycles of $95{ }^{\circ} \mathrm{C}$ for $1 \mathrm{~min}, 50^{\circ} \mathrm{C}$ for $1 \mathrm{~min}$, and $72{ }^{\circ} \mathrm{C}$ for $1 \mathrm{~min}$ and then $72{ }^{\circ} \mathrm{C}$ for $7 \mathrm{~min}$. The $K 2 \mathrm{~A}$ gene, which is consistent with the K2 serotype, was detected by PCR. PCR was also positive for the virulenceassociated gene $r m p A$. To assess for the presence of hypermucoviscosity, a string test was performed on the organism grown in $5 \%$ sheep blood agar. The formation of a mucous string of $>5 \mathrm{~mm}$ in length after touching a colony with a loop was considered positive [9]. The isolated $K$. pneumoniae had a positive string test consistent with a hypermucoviscous phenotype (Fig. 2).

\section{Discussion}

We present the case of invasive liver abscess syndrome with endogenous endophthalmitis caused by the K2 serotype of $K$. pneumoniae extending to complete ablepsia despite improvement of the pathophysiology of severe sepsis. One systematic review reported $K$. pneumoniae had been the most common causative organism (27 \%) in endogenous bacterial endophthalmitis. Following other organisms were Staphylococcus aureus (10 \%), Pseudomonas aeruginosa (6\%), Group B streptococci (6\%), and Neisseria meningitidis (5\%) [10]. Therefore, use of broadspectrum antibiotics must be considered until identification of causative microorganism.

This case of a primary liver abscess caused by the definite K2 serotyping of $K$. pneumoniae is the first adult case in Japan. This invasive syndrome caused by K. pneumoniae serotypes $\mathrm{K} 1$ or $\mathrm{K} 2$ has been reported mainly in Southeast Asian countries, especially in Taiwan. We found around 50 cases with liver abscess caused by K. pneumoniae serotype $\mathrm{K} 2$ all over the world [11, 12]. However, in 


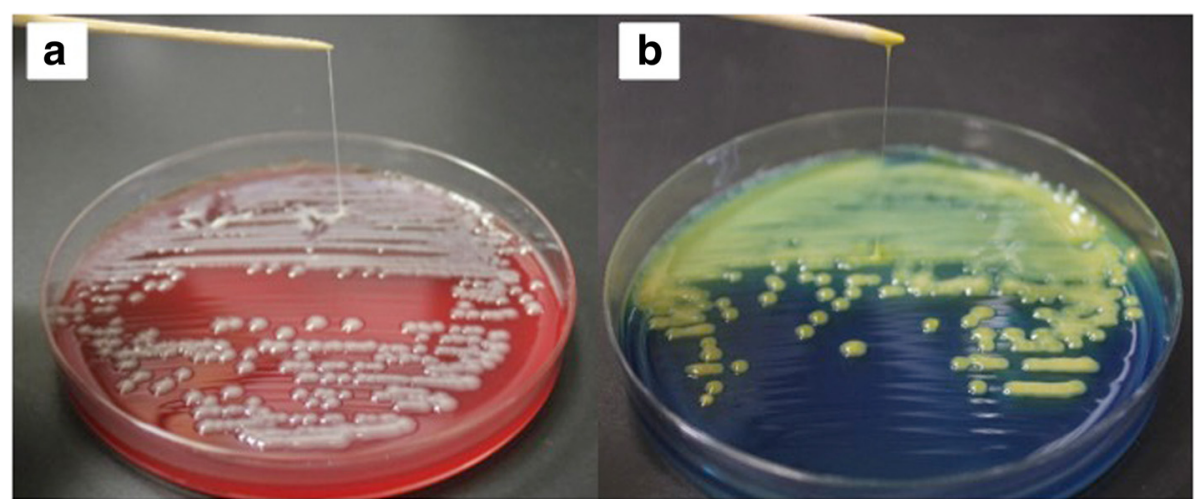

Fig. 2 String test. Stretching colonies form a string of $>5 \mathrm{~mm}$ in length in sheep blood agar (a) and in bromothymol blue lactose agar (b)

Japan, only three cases-two in elderly men infected with the $\mathrm{K} 1$ serotype and one in a 7-year-old child with the K2 serotype-have been reported [4-6], when we searched PubMed and the Igaku Chuo Zasshi databases for papers published between Jun 01, 1970, and Dec 31, 2015, by using combinations of the following keywords: "Klebsiella pneumoniae," "liver abscess," "K1" or "K2," except minutes and selected articles about this invasive syndrome published by Japanese authors.

Almost 30 cases of primary liver abscess caused by $K$. pneumoniae with endogenous endophthalmitis have been reported in Japan (all reports in Japanese); we conducted a search of the medical literature published using the Igaku Chuo Zasshi database and "liver abscess," "endophthalmitis," and "Klebsiella pneumoniae" as search terms. In addition, we could find two more Japanese literatures related to invasive liver abscess syndrome when we searched Igaku Chuo Zasshi database by using following keywords: "Klebsiella pneumoniae," "liver abscess," and "rmpA" [13, 14]. The K1 and K2 serotype were suspected in all cases because of the clinical features, but examination of the serotype has never been reported. Therefore, an invasive liver abscess caused by the $\mathrm{K} 1$ or $\mathrm{K} 2$ serotype of $K$. pneumoniae may not be rare in Japan.

The virulence-associated gene $\operatorname{rmp} A$ was positive in our case. rmpA is not an independent factor contributing to a liver abscess, but it aids in capsule synthesis. One report showed that all $K$. pneumoniae strains that cause liver abscesses and abscesses at other sites are rmpA-positive. rmpA has been confirmed as a gene that regulates capsular polysaccharide synthesis [15]. Thus, in our case, rmpA was probably associated with liver abscess formation.

\section{Conclusions}

Although a primary liver abscess caused by $K$. pneumoniae with a hypermucoviscous phenotype is infrequently reported outside Southeast Asia, physicians should recognize this syndrome, and appropriate diagnosis and treatment is essential for both saving patients' lives and preserving organ function, especially for visual acuity.

\section{Consent}

Written informed consent was obtained from the patient for publication of this case report and any accompanying images. A copy of the written consent form is available for review by the Editor-in-Chief of this journal.

\section{Abbreviations \\ K. pneumonia, Klebsiella pneumonia; PCR, polymerase chain reaction; rmpA, mucoid phenotype A gene}

\section{Competing interests}

The authors declare that they have no competing interests.

\section{Authors' contributions}

RS drafted the manuscript. DK drafted the manuscript and contributed to the conception and design of the case report. YG, HY, TA, and MK carried out the molecular genetic studies and helped to draft the manuscript. TO and SI helped to draft the manuscript. SK contributed to the conception and design of the case report and critically revised the draft for important intellectual content. All of the authors read and approved the final manuscript.

\section{Author details}

${ }^{1}$ Department of Emergency and Critical Care Medicine, Tohoku University Hospital, 1-1 Seiryo-machi, Aoba-ku, Sendai 980-8574, Japan. ²Department of Surgery, Yamagata Prefectural Central Hospital, 1800 Aoyagi, Yamagata 990-2292, Japan. ${ }^{3}$ Division of Emergency and Critical Care Medicine, Tohoku University Graduate School of Medicine, 2-1 Seiryo-machi, Aoba-ku, Sendai 980-8575, Japan. ${ }^{4}$ Division of Infection Control and Laboratory Diagnostics, Tohoku University Graduate School of Medicine, 2-1 Seiryo-machi, Aoba-ku, Sendai 980-8575, Japan. ${ }^{5}$ Department of Microbiology and Infectious Diseases, Nara Medical University, 840 Shijo-machi, Kashihara 634-8521, Japan. ${ }^{6}$ Department of Neurology, Tohoku University Graduate School of Medicine, 2-1 Seiryo-machi, Aoba-ku, Sendai 980-8575, Japan.

Received: 13 April 2016 Accepted: 12 July 2016

Published online: 25 July 2016

\section{References}

1. Chang FY, Chou MY, Fan RL, Shaio MF. A clinical study of Klebsiella liver abscess. Taiwan Yi Xue Hui Za Zhi. 1988;87:282-7.

2. Siu LK, Yeh KM, Lin JC, Fung CP, Chang FY. Klebsiella pneumoniae liver abscess: a new invasive syndrome. Lancet Infect Dis. 2012;12:881-7.

3. Yeh KM, Lin JC, Yin FY, Fung CP, Hung HC, Siu LK, et al. Revisiting the importance of virulence determinant magA and its surrounding genes in 
Klebsiella pneumoniae causing pyogenic liver abscesses: exact role in serotype K1 capsule formation. J Infect Dis. 2010;201:1259-67.

4. Kohayagawa Y, Nakao K, Ushita M, Niino N, Koshizaki M, Yamamori Y, et al. Pyogenic liver abscess caused by Klebsiella pneumoniae genetic serotype K1 in Japan. J Infect Chemother. 2009;15:248-51.

5. Maruno T, Ooiwa Y, Takahashi K, Kodama Y, Takakura S, Ichiyama S, et al. A liver abscess deprived a healthy adult of eyesight: endogenous endophthalmitis associated with a pyogenic liver abscess caused by serotype K1 Klebsiella pneumonia. Intern Med. 2013;52:919-22.

6. Yoshimura A, Matsubayashi T, Matsubayashi R. Liver abscess caused by Klebsiella pneumoniae serotype K2 in a child. J Pediat Infect Dis and Immun. 2012;24:275-8.

7. Yu WL, Fung CP, Ko WC, Cheng KC, Lee CC, Chuang YC. Polymerase chain reaction analysis for detecting capsule serotypes K1 and K2 of Klebsiella pneumoniae causing abscesses of the liver and other sites. J Infect Dis. 2007;195:1235-6

8. Siu LK, Fung CP, Chang FY, Lee N, Yeh KM, Koh TH, et al. Molecular typing and virulence analysis of serotype K1 Klebsiella pneumoniae strains isolated from liver abscess patients and stool samples from noninfectious subjects in Hong Kong, Singapore, and Taiwan. J Clin Microbiol. 2011;49:3761-5.

9. Fang $C T$, Chuang YP, Shun $C T$, Chang SC, Wang JT. A novel virulence gene in Klebsiella pneumoniae strains causing primary liver abscess and septic metastatic complications. J Exp Med. 2004;199:697-705.

10. Jackson TL, Paraskevopoulos T, Georgalas I. Systematic review of 342 cases of endogenous bacterial endophthalmitis. Surv Ophthalmol. 2014;59:627-35.

11. Lin JC, Koh TH, Lee N, Fung CP. Chang FY, Tsai YK, et al. Genotypes and virulence in serotype K2 Klebsiella pneumoniae from liver abscess and non-infectious carriers in Hong Kong, Singapore and Taiwan. Gut Pathog. 2014;6:21.

12. Fang CT, Lai SY, Yi WC, Hsueh PR, Liu KL, Chang SC. Klebsiella pneumoniae genotype K1: an emerging pathogen that causes septic ocular or central nervous system complications from pyogenic liver abscess. Clin Infect Dis. 2007:45:284-93.

13. Nakamoto K, Koide S, Nagatomo T, Higaki M, Wada H, Ishii H, et al. Severe primary liver abscess and Septic pulmonary embolism due to Klebsiella pneumoniae with hypermucoviscosity phenotype. Kansenshogaku Zasshi. 2011:85:366-9.

14. Yanagi K, Suzuki K, Kase M, Shimizu M, Tanaka S, Aoki C, et al. A case of type 2 diabetes mellitus associated with severe multi-focal infection caused by Klebsiella pneumoniae. J Jpn Diab Soc. 2014;57:28-34.

15. Yu WL, Ko WC, Cheng KC, Lee HC, Ke DS, Lee CC, et al. Association between rmpA and magA genes and clinical syndromes caused by Klebsiella pneumoniae in Taiwan. Clin Infect Dis. 2006;42:1351-8.

\section{Submit your manuscript to a SpringerOpen ${ }^{\circ}$ journal and benefit from:}

- Convenient online submission

- Rigorous peer review

- Immediate publication on acceptance

- Open access: articles freely available online

- High visibility within the field

Retaining the copyright to your article 